\title{
Fatores de risco para baixa adesão ao tratamento farmacológico de hipertensão arterial sistêmica na Atenção Primária à Saúde
}

\author{
Risk factors for low adherence to the pharmacological treatment of systemic arterial \\ hypertension in Primary Health Care
}

Factores de riesgo de baja adherencia al tratamiento farmacológico de la hipertensión arterial sistémica en Atención Primaria de Salud

João Ricardo Arraes Oliveira ${ }^{1 *}$, Marcello Augusto Anchieta Santos Filho², Maria Júlia Moura Nascimento Santos ${ }^{1}$, Thayná Oliveira Militão ${ }^{3}$, Árgila Gonçalves de Carvalho Santana ${ }^{3}$.

\section{RESUMO}

Objetivo: Analisar na literatura científica os fatores de risco para baixa adesão ao tratamento farmacológico de hipertensão arterial sistêmica (HAS) em pacientes atendidos em centros de atenção primária à saúde (APS). Métodos: Trata-se de uma revisão de literatura integrativa de caráter descritivo, realizada através da pesquisa de artigos científicos indexados nos portais PubMed, BVS e SciELO. Resultados: A pesquisa nas bases de dados resultou na seleção de 18 artigos, obedecendo aos critérios de inclusão e exclusão estabelecidos. Constatou-se que os principais fatores de risco para a baixa adesão medicamentosa são aqueles de cunho social, clínico e psicológico, destacando-se também elementos relacionados aos serviços de saúde. Considerações finais: A baixa adesão ao tratamento farmacológico da HAS pode ser favorecida por uma grande variedade e heterogeneidade de elementos, o que impacta negativamente o controle da doença no contexto da APS. Os fatores de risco identificados são relevantes para a elaboração de estudos posteriores que possibilitem o planejamento e execução de intervenções efetivas.

Palavras-chave: Cooperação e adesão ao tratamento, Hipertensão, Atenção primária à saúde.

\begin{abstract}
Objective: To analyze in scientific literature the risk factors for low adherence to the pharmacological treatment of systemic arterial hypertension (SAH) in patients treated at primary health care centers (PHC). Methods: This is an integrative literature review of descriptive character, carried out through the search for scientific articles indexed in the PubMed, BVS and SciELO portals. Results: The database search resulted in the selection of 18 articles, in accordance to the established inclusion and exclusion criteria. It was found that the main risk factors for low medication adherence are those of social, clinical and psychological nature, while also standing out elements related to the health services. Final considerations: Low adherence to the pharmacological treatment of SAH can be favored by a wide variety and heterogeneity of elements, which negatively impacts the control of the disease in the context of PHC. The identified risk factors are relevant for the preparation of further studies that enable the planning and execution of effective actions.
\end{abstract}

Keywords: Treatment adherence and compliance, Hypertension, Primary health care.

\section{RESUMEN}

Objetivo: Analizar en la literatura científica los factores de riesgo de baja adherencia al tratamiento farmacológico de la hipertensión arterial sistémica (HAS) en pacientes atendidos en centros de atención

1 Universidade de Pernambuco (UPE), Recife - PE. *E-mail: joao.arraes@upe.br

2 Universidade Federal de Sergipe (UFS), Aracaju - SE.

${ }^{3}$ Universidade Federal do Recôncavo da Bahia (UFRB), Cruz das Almas - BA.

SUBMETIDO EM: 10/2020

ACEITO EM: 11/2020

PUBLICADO EM: 1/2021 
primaria de salud (APS). Métodos: Se trata de una revisión bibliográfica integradora de carácter descriptivo, realizada a través de la búsqueda de artículos científicos indexados en los portales PubMed, BVS y SciELO. Resultados: La búsqueda en las bases de datos resultó en la selección de 18 artículos, de acuerdo con los criterios de inclusión y exclusión establecidos. Se encontró que los principales factores de riesgo para baja adherencia a la medicación son los de naturaleza social, clínica y psicológica, destacandose también elementos relacionados con los servicios de salud. Consideraciones finales: Baja adherencia al tratamiento farmacológico de la HAS puede verse favorecida por una amplia variedad y heterogeneidad de elementos, lo que impacta negativamente en el control de la enfermedad en el contexto de la APS. Los factores de riesgo identificados son relevantes para la elaboración de nuevos estudios que permitan la planificación y ejecución de acciones efectivas.

Palabras clave: Cumplimiento y adherencia al tratamiento, Hipertensión, Atención primaria de salud.

\section{INTRODUÇÃO}

A hipertensão arterial sistêmica (HAS) é uma entidade nosológica de etiologia multifatorial caracterizada pela elevação persistente da pressão arterial em níveis iguais ou superiores a $140 \mathrm{mmHg}$ ou $90 \mathrm{mmHg}$, para pressão sistólica e diastólica, respectivamente. Trata-se de uma doença crônica que, a despeito de sua evolução insidiosa, pode atuar como fator precipitante de uma grande variedade de afecções sistêmicas severas e potencialmente fatais, tais como acidente vascular encefálico (AVE), infarto agudo do miocárdio (IAM), insuficiência cardíaca (IC), doença renal crônica (DRC), entre outros. Muitos dos fatores de risco para o desenvolvimento da patologia, como o sedentarismo e os maus hábitos alimentares, estão intrinsecamente relacionados ao estilo de vida dos indivíduos; isso, por sua vez, justifica a frequente apresentação concomitante de demais desordens metabólicas em pacientes hipertensos, como dislipidemia, obesidade e diabetes mellitus (MAGRINI DW e MARTINI JG, 2012; MALACHIAS MVB, et al., 2016).

A HAS é considerada um problema de saúde pública em vários países, acometendo cerca de $25 \%$ da população mundial (CORREIA BR, et al., 2017). No Brasil, estima-se que a HAS acometa mais de 30 milhões de pessoas, sendo que a prevalência da enfermidade nas cidades brasileiras situa-se em torno de $32,5 \%$, para indivíduos adultos, e de $60 \%$, para indivíduos idosos; esses dados mostram-se particularmente relevantes quando se considera que a patologia constitui um dos principais fatores de risco para o desenvolvimento de doenças cardiovasculares, as quais representam a maior causa de mortalidade no país, bem como um ônus econômico para o sistema de saúde nacional (MALACHIAS MVB, et al., 2016; SILVA MLB e BOUSFIELD ABS, 2016).

A conduta terapêutica para o controle da HAS deve levar em consideração fatores de risco e aspectos clínicos dos pacientes. Drogas atualmente utilizadas no manejo da doença incluem diuréticos, bloqueadores dos receptores AT1 da angiotensina (BRAs), inibidores da enzima conversora da angiotensina (IECAs) e betabloqueadores, algumas das quais frequentemente são empregadas em associação (MALACHIAS MVB, et al., 2016).

A despeito da comprovada eficácia terapêutica das medicações supracitadas, a não adesão ao tratamento prevalece como um impasse no controle da HAS. Estima-se que apenas $1 / 3$ dos pacientes hipertensos apresentem um controle satisfatório dos níveis pressóricos (REINERS AAO, et al., 2013; SILVA MLB e BOUSFIELD ABS, 2016). A não adesão ao tratamento farmacológico é definida como o abandono ou a irregularidade do uso das medicações receitadas pelo profissional de saúde. A cronicidade da HAS, associada ao caráter usualmente assintomático da patologia, justificam em grande parte as baixas taxas de aderência dos pacientes aos tratamentos medicamentosos propostos.

Soma-se a esses elementos o fato de os esquemas terapêuticos necessários para o efetivo controle da doença muitas vezes incluírem associações de variados fármacos distintos, requerendo um elevado grau de organização e de disciplina por parte dos pacientes, o que pode prejudicar substancialmente a adesão dos mesmos à terapia (JORNADA BA, et al., 2012; GEWEHR DM, et al., 2018; MOURA AA, et al., 2016). Sendo guiada pelos preceitos de longitudinalidade, de comunicação e de responsabilização, a Atenção Primária à 
Saúde (APS) desempenha um papel crucial no acompanhamento e tratamento dos pacientes portadores de HAS, e consequentemente, na redução das taxas de agravos secundários à enfermidade.

Diante da elevada incidência de HAS e, sobretudo, das baixas taxas de controle da doença por parte dos pacientes, nova medidas gerenciais e estratégias têm sido elaboradas pelos profissionais da APS, visando aprimorar o papel da equipe de saúde na promoção da saúde ao paciente hipertenso. Nesse contexto, conhecer o perfil clínico e sociodemográfico daqueles pacientes que podem estar mais propensos à baixa adesão terapêutica é fundamental para possibilitar a adequada elaboração de estratégias de intervenção clínica, terapêutica e educacional (DANTAS RCO e RONCALLI AG, 2019; REINERS AAO, et al., 2013).

Estudos sobre a temática fazem-se relevantes para proporcionar o embasamento teórico que possibilite um direcionamento dos profissionais de saúde acerca das atitudes e estratégias que devem ser contempladas ao lidar-se com o grupo de pacientes portadores de HAS. Diante do exposto, o presente trabalho tem como objetivo analisar na literatura científica os fatores de risco para baixa adesão ao tratamento farmacológico de HAS em pacientes atendidos em centros de APS.

\section{MÉTODOS}

O presente estudo se trata de uma revisão de literatura de caráter descritivo que visa responder a seguinte pergunta norteadora: "Quais são os fatores de risco para a baixa adesão ao tratamento de HAS no contexto da APS?". A revisão foi realizada com base nos artigos científicos publicados em periódicos indexados nos portais PubMed, SciELO e BVS.

Para a pesquisa nas bases de dados científicas, foi utilizada como estratégia de busca a combinação dos Descritores em Ciências da Saúde (DeCS) "Hypertension", "Treatment Adherence and Compliance" e "Primary Health Care", conectados pelo Operador Booleano "AND". A busca utilizou o filtro temporal para seleção de trabalhos publicados entre os anos de 2015 e 2020. A pesquisa foi realizada no período de agosto a setembro de 2020.

Foi definido o seguinte critério de inclusão: artigos científicos de qualquer idioma, sem restrições em relação a país ou região de publicação, que avaliassem a associação de variáveis de qualquer natureza ao nível de adesão ao tratamento de HAS em pacientes atendidos na APS.

Como critérios de exclusão, foram estabelecidos: revisões de literatura narrativas, integrativas ou sistemáticas; estudos de caso; protocolos de pesquisa; estudos que não avaliassem pacientes da APS; trabalhos cujo texto completo não estivesse disponível na íntegra; artigos cuja temática não contemplasse o objetivo deste estudo.

A seleção dos trabalhos ocorreu através da análise do título/resumo dos artigos recuperados com a estratégia de busca estabelecida, após a remoção das duplicatas. Os artigos que se adequaram aos critérios seletivos desta revisão passaram por uma leitura crítica, com o objetivo de identificar fatores relacionados à baixa adesão terapêutica em pacientes hipertensos atendidos da APS.

Em seguida, esses fatores foram analisados, agrupados em um quadro teórico e discutidos de acordo com a ênfase abordada. Conforme a resolução no 466/2012 do Conselho Nacional de Saúde, não foi necessária a apreciação do Comitê de Ética em Pesquisa (CEP) por se tratar de uma revisão de literatura integrativa.

\section{RESULTADOS}

A etapa de busca dos trabalhos nas bases de dados possibilitou a recuperação de 643 artigos. Desses, 18 foram considerados elegíveis para compor esta revisão em virtude de satisfazerem os critérios de inclusão e exclusão pré-definidos (Figura 1). A amostra final de artigos incluiu: 1 publicação de 2020; 1 publicação de 2019; 2 publicações de 2018; 6 publicações de 2017; 3 publicações de 2016; e 5 publicações de 2015. No que diz respeito ao idioma dos artigos, foram identificados: 6 publicações de língua portuguesa; 8 publicações de língua inglesa; e 4 publicações de língua espanhola. 
Figura 1 - Fluxograma do processo de identificação, triagem e inclusão de artigos das bases de dados PubMed, SciELO e BVS.
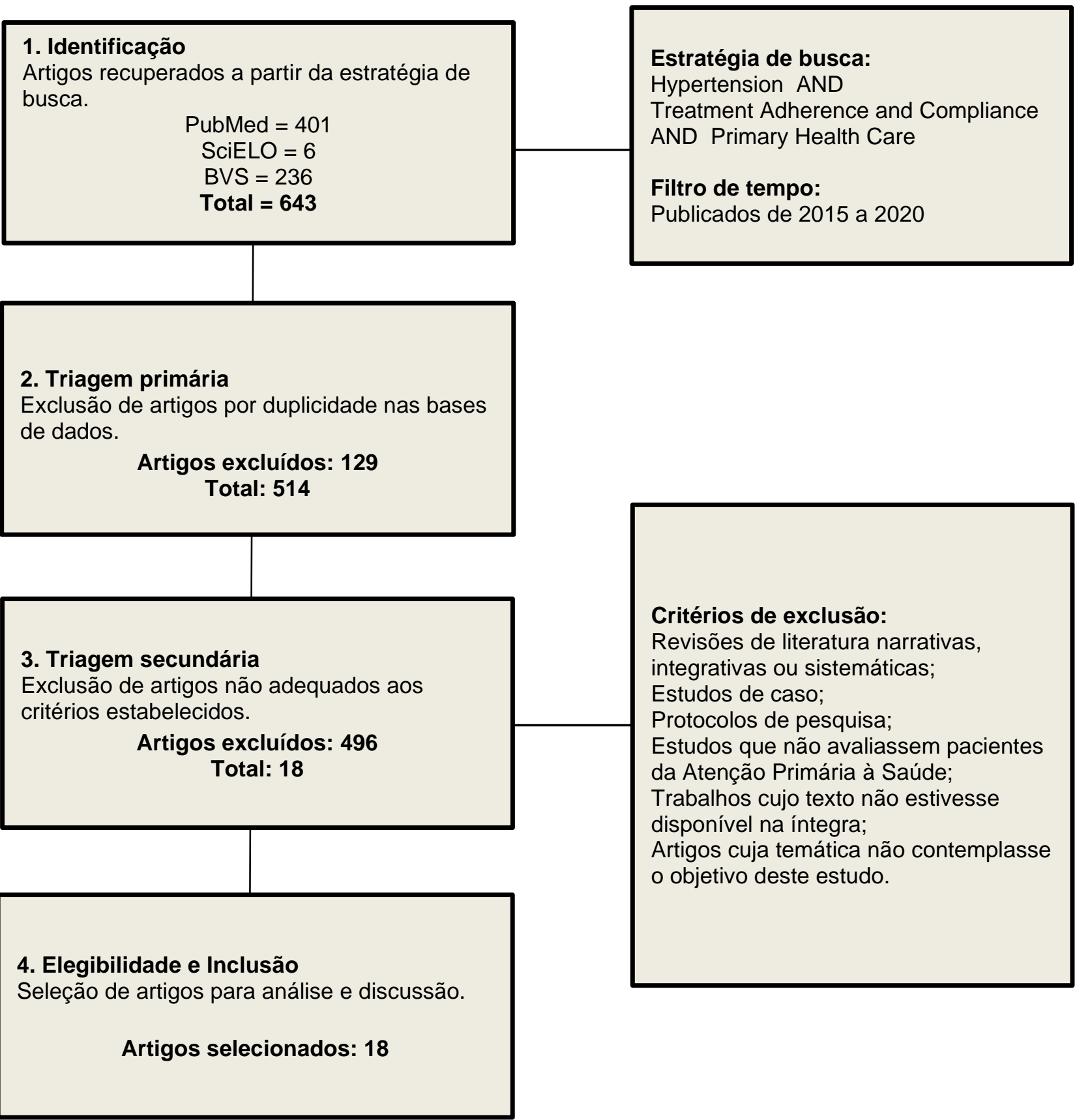

\section{Triagem primária}

Exclusão de artigos por duplicidade nas bases

Artigos excluídos: 129

Total: $\mathbf{5 1 4}$

\section{Triagem secundária}

Exclusão de artigos não adequados aos

Artigos excluídos: 496

Total: 18

Fonte: Oliveira JRA, et al., 2020.

A partir dos artigos selecionados, foram extraídas informações referentes a título, autor/ano, periódico e fatores relacionados à baixa adesão ao tratamento de HAS identificados. Esses dados foram agrupados para facilitar a visualização e compreensão das informações (Quadro 1) (Quadro 2) (Quadro 3). 
Quadro 1 - Caracterização dos estudos selecionados conforme título; autor-ano; periódico e fatores relacionados à baixa adesão terapêutica de hipertensão arterial sistêmica.

\begin{tabular}{|c|c|c|c|c|}
\hline $\mathbf{N}$. & Título & Autor/ano & Periódico & $\begin{array}{c}\text { Fatores relacionados à baixa adesão ao tratamento } \\
\text { de HAS identificados }\end{array}$ \\
\hline A1 & $\begin{array}{l}\text { The outpatient management of } \\
\text { hypertension at two Sierra Leonean health } \\
\text { centres: A mixed-method investigation of } \\
\text { follow-up compliance and patient-reported } \\
\text { barriers to care. }\end{array}$ & Herskind J, et al. (2020) & $\begin{array}{l}\text { African Journal of } \\
\text { Primary Health } \\
\text { Care \& Family } \\
\text { Medicine. }\end{array}$ & $\begin{array}{l}\text { Falta de acesso à transporte; } \\
\text { Dificuldades financeiras; } \\
\text { Conflitos de agendamento. }\end{array}$ \\
\hline $\mathrm{A} 2$ & $\begin{array}{c}\text { Adesão ao tratamento e hábitos de vida de } \\
\text { hipertensos. }\end{array}$ & Dallacosta FM, et al. (2019) & $\begin{array}{l}\text { Revista Online de } \\
\text { Pesquisa Cuidado } \\
\text { é Fundamental. }\end{array}$ & $\begin{array}{l}\text { Dificuldade de listar a medicação em uso; } \\
\text { Ler, abrir e lembrar de tomar a medicação. }\end{array}$ \\
\hline A3 & $\begin{array}{l}\text { Medication adherence improvement similar } \\
\text { for shared decision-making preference or } \\
\text { longer patient-provider relationship }\end{array}$ & Schoenthaler A, et al. (2018) & $\begin{array}{l}\text { Journal of the } \\
\text { American Board of } \\
\text { Family Medicine. }\end{array}$ & $\begin{array}{l}\text { Ausência de decisão ativa ou compartilhada dos } \\
\text { pacientes com os profissionais de saúde; } \\
\text { Menor duração da relação médico-paciente. }\end{array}$ \\
\hline A4 & $\begin{array}{l}\text { Dificuldades de idosos na adesão ao } \\
\text { tratamento da hipertensão arterial. }\end{array}$ & Resende AKM, et al. (2018) & $\begin{array}{l}\text { Revista de } \\
\text { Enfermagem da } \\
\text { UFPE online. }\end{array}$ & $\begin{array}{c}\text { Medo de interações medicamentosas; } \\
\text { Esquecimento; } \\
\text { Falta de apoio social/familiar }\end{array}$ \\
\hline A5 & $\begin{array}{l}\text { Predictors of medication adherence and } \\
\text { blood pressure control among Saudi } \\
\text { hypertensive patients attending Primary } \\
\text { Care Clinics: A Cross-sectional study. }\end{array}$ & Khayyat, SM, et al. (2017) & PLOS ONE. & $\begin{array}{c}\text { Sexo feminino; } \\
\text { Idade inferior a } 65 \text { anos; } \\
\text { Ausência de diabetes mellitus. }\end{array}$ \\
\hline A6 & $\begin{array}{l}\text { Addressing the social needs of } \\
\text { hypertensive patients: the role of patient- } \\
\text { provider communication as a predictor of } \\
\text { medication adherence }\end{array}$ & Schoenthaler A, et al. (2017) & $\begin{array}{l}\text { Circulation: } \\
\text { Cardiovascular } \\
\text { Quality and } \\
\text { Outcomes. }\end{array}$ & $\begin{array}{l}\text { Pacientes negros, portadores de diabetes mellitus e } \\
\text { usuários de anti-hipertensivos não IECAs (inibidores } \\
\text { da enzima conversora de angiotensina); Ausência de } \\
\text { discussões sobre as condições sociodemográficas dos } \\
\text { pacientes e sobre o tratamento nas consultas clínicas; } \\
\text { Consultas clínicas não centradas nos pacientes. }\end{array}$ \\
\hline
\end{tabular}

Fonte: Oliveira JRA et al., 2020.

REAS/EJCH | Vol.13(1) | e5514 | DOI: https://doi.org/10.25248/reas.e5514.2021 Página 5 de 11 
Quadro 2 - Caracterização dos estudos selecionados conforme título; autor-ano; periódico e fatores relacionados à baixa adesão terapêutica de hipertensão arterial sistêmica.

\begin{tabular}{|c|c|c|c|c|}
\hline $\mathbf{N}$. & Título & Autor/ano & Periódico & $\begin{array}{l}\text { Fatores relacionados à baixa adesão ao } \\
\text { tratamento de HAS identificados }\end{array}$ \\
\hline A7 & $\begin{array}{l}\text { Adesão ao tratamento da } \\
\text { hipertensão arterial entre usuários } \\
\text { da Estratégia Saúde da Família } \\
\text { em um município do Piauí }\end{array}$ & $\begin{array}{l}\text { Rocha MLF, } \\
\text { et al. (2017) }\end{array}$ & Revista de APS - Atenção Primária à Saúde. & $\begin{array}{c}\text { Uso de bebidas alcoólicas; } \\
\text { Alimentação rica em gorduras/frituras; } \\
\text { Utilização de medicamentos múltiplas vezes ao } \\
\text { dia e ingestão de mais de } 2 \text { comprimidos } \\
\text { simultaneamente. }\end{array}$ \\
\hline A8 & $\begin{array}{l}\text { Assessing adherence to } \\
\text { antihypertensive therapy in } \\
\text { Primary Health Care in Namibia: } \\
\text { findings and implications. }\end{array}$ & $\begin{array}{l}\text { Nashilongo } \\
\text { MM, et al. } \\
(2017)\end{array}$ & Cardiovascular Drugs and Therapy. & $\begin{array}{l}\text { Ausência de suporte social ao tratamento; } \\
\text { Falta a consultas clínicas agendadas; } \\
\text { Comparecimento irregular às consultas de } \\
\text { acompanhamento. }\end{array}$ \\
\hline A9 & $\begin{array}{c}\text { Dificuldades de adesão ao } \\
\text { tratamento por hipertensos de } \\
\text { uma unidade de atenção primária } \\
\text { à saúde }\end{array}$ & $\begin{array}{l}\text { Becho AS, et } \\
\text { al. (2017) }\end{array}$ & Revista de APS - Atenção Primária à Saúde. & $\begin{array}{c}\text { Ausência de conhecimentos sobre patologia e } \\
\text { tratamento não-medicamentoso; } \\
\text { Ausência de sintomas; } \\
\text { Efeitos colaterais da terapia; } \\
\text { Dificuldades financeiras. }\end{array}$ \\
\hline A10 & $\begin{array}{l}\text { Relationship between medication } \\
\text { adherence and health beliefs } \\
\text { among patients with hypertension } \\
\text { in Oman: Pilot study }\end{array}$ & $\begin{array}{l}\text { Al-Noumani } \\
\text { H, et al. } \\
(2017)\end{array}$ & Sultan Qaboos University Medical Journal. & $\begin{array}{l}\text { Baixa autoeficácia psicológica; } \\
\text { Crenças mais fracas sobre a importância dos } \\
\text { medicamentos anti-hipertensivos. }\end{array}$ \\
\hline A11 & $\begin{array}{l}\text { Adherencia terapéutica y } \\
\text { creencias sobre su salud en } \\
\text { pacientes hipertensos }\end{array}$ & $\begin{array}{l}\text { Pérez- } \\
\text { Rosabal E, } \\
\text { et al. (2016) }\end{array}$ & MEDISAN. & $\begin{array}{l}\text { Falta de percepção acerca da susceptibilidade } \\
\text { e gravidade das complicações da hipertensão; } \\
\text { Falta de percepção acerca dos benefícios do } \\
\text { tratamento anti-hipertensivo. }\end{array}$ \\
\hline A12 & $\begin{array}{c}\text { Factores de no adhesión al } \\
\text { tratamiento de la hipertensión } \\
\text { arterial }\end{array}$ & $\begin{array}{l}\text { Moura AA, et } \\
\text { al. (2016) }\end{array}$ & Enfermería Global. & $\begin{array}{c}\text { Estresse; } \\
\text { Alterações no padrão de sono; } \\
\text { Dificuldades de adaptação às mudanças no } \\
\text { estilo de vida. }\end{array}$ \\
\hline
\end{tabular}

Fonte: Oliveira JRA et al., 2020.

REAS/EJCH | Vol.13(1) | e5514 | DOI: https://doi.org/10.25248/reas.e5514.2021 Página 6 de 11 
Quadro 3 - Caracterização dos estudos selecionados conforme título; autor-ano; periódico e fatores relacionados à baixa adesão terapêutica de hipertensão arterial sistêmica (conclusão).

\begin{tabular}{|c|c|c|c|c|}
\hline $\mathbf{N}$. & Título & Autor/ano & Periódico & $\begin{array}{l}\text { Fatores relacionados à baixa adesão ao } \\
\text { tratamento de HAS identificados }\end{array}$ \\
\hline A13 & $\begin{array}{c}\text { Perceived social standing, } \\
\text { medication nonadherence, and } \\
\text { systolic blood pressure in the } \\
\text { Rural South. }\end{array}$ & $\begin{array}{l}\text { Cummings } \\
\text { DM, et al. } \\
(2016)\end{array}$ & The Journal of Rural Health. & $\begin{array}{c}\text { Idade mais jovem; } \\
\text { Raça afro-americana; } \\
\text { Menor posição social percebida. }\end{array}$ \\
\hline A14 & $\begin{array}{l}\text { Predictors of non-adherence to } \\
\text { antihypertensive medication in } \\
\text { Kinshasa, Democratic Republic of } \\
\text { Congo: a cross-sectional study. }\end{array}$ & $\begin{array}{l}\text { Lulebo AM, } \\
\text { et al. (2015) }\end{array}$ & BMC Research Notes. & $\begin{array}{l}\text { Pouco conhecimento das complicações da } \\
\text { hipertensão; } \\
\text { Indisponibilidade de anti-hipertensivos nas } \\
\text { unidades de saúde; } \\
\text { Falta de educação em saúde para hipertensos } \\
\text { nas unidades de saúde }\end{array}$ \\
\hline A15 & $\begin{array}{c}\text { Prevalência de não adesão à } \\
\text { farmacoterapia anti-hipertensiva e } \\
\text { fatores associados }\end{array}$ & $\begin{array}{l}\text { Barreto MS, } \\
\text { et al. (2015) }\end{array}$ & Revista Brasileira de Enfermagem. & $\begin{array}{c}\text { Pacientes não brancos; } \\
\text { Menos de oito anos de estudo; } \\
\text { Não frequentar consultas médicas; } \\
\text { Utilizar mais de duas medicações anti- } \\
\text { hipertensivas; Não possuir plano de saúde. }\end{array}$ \\
\hline A16 & $\begin{array}{l}\text { Prevalencia y determinantes de } \\
\text { adherencia a terapia } \\
\text { antihipertensiva en pacientes de } \\
\text { la Región Metropolitana }\end{array}$ & $\begin{array}{l}\text { Varleta } P \text {, et } \\
\text { al. (2015) }\end{array}$ & Revista médica de Chile. & $\begin{array}{c}\text { Idade menos elevada; } \\
\text { Não estar desempregado; } \\
\text { Esquecimento de tomar os comprimidos; } \\
\text { Efeitos adversos. }\end{array}$ \\
\hline A17 & $\begin{array}{l}\text { Adesão ao uso de medicamentos } \\
\text { entre idosos hipertensos }\end{array}$ & $\begin{array}{l}\text { Aiolfi CR, et } \\
\text { al. (2015) }\end{array}$ & Revista Brasileira de Geriatria e Gerontologia. & $\begin{array}{l}\text { Idosos mais jovens; } \\
\text { Idosos que moram desacompanhados. }\end{array}$ \\
\hline A18 & $\begin{array}{l}\text { Evaluación del control de la } \\
\text { presión arterial y la adherencia } \\
\text { terapéutica en hipertensos } \\
\text { seguidos en el Programa de } \\
\text { Salud Cardiovascular (PSCV) }\end{array}$ & $\begin{array}{l}\text { Chacón J, et } \\
\text { al. (2015) }\end{array}$ & Revista chilena de cardiologia. & $\begin{array}{c}\text { Sexo masculino; } \\
\text { Baixa renda familiar; } \\
\text { Baixa escolaridade; } \\
\text { Relação médico-paciente inadequada; } \\
\text { Alto nível de estresse emocional; Depressão. }\end{array}$ \\
\hline
\end{tabular}

Fonte: Oliveira JRA et al., 2020.

REAS/EJCH | Vol.13(1) | e5514 | DOI: https://doi.org/10.25248/reas.e5514.2021 Página 7 de 11 


\section{DISCUSSÃO}

A partir da leitura crítica e da comparação dos artigos selecionados, foi possível constatar que um espectro bastante heterogêneo de fatores psicológicos, sociais, clínicos e ambientais podem exercer um impacto negativo na aderência ao tratamento medicamentoso de HAS nos pacientes que são atendidos em clínicas de APS.

Para uma melhor discussão das informações extraídas dos artigos, os fatores de risco para a baixa adesão terapêutica foram categorizados da seguinte forma: 1) Fatores associados às condições sociodemográficas; 2) Fatores associados a condições clínicas, hábitos de vida e outras características intrínsecas aos pacientes; 3) Fatores associados a Unidades e Equipes de Saúde.

\section{Fatores associados às condições sociodemográficas}

Em relação aos fatores de risco sociodemográficos para a baixa adesão ao tratamento da HAS, destacamse: pele não branca, utilização exclusiva do sistema de saúde público, falta de acesso a transporte, baixa renda e baixo nível de escolaridade (BARRETO MS, et al., 2015; CHACÓN J, et al., 2015; CUMMINGS DM, et al., 2016; HERSKIND J, et al., 2020; SCHOENTHALER A, et al., 2017; KHAYYAT SM, et al., 2017).

Muitos dos fatores supracitados são indicativos de baixo nível socioeconômico, o que comumente faz parte da realidade das populações atendidas na APS; é imprescindível avaliar o estado de vulnerabilidade social dos pacientes, uma vez que esse pode constituir um fator determinante para a baixa adesão aos medicamentos (SCHOENTHALER A, et al., 2017).

Quanto à influência do sexo na adesão terapêutica, a literatura é divergente, com a maioria das pesquisas reportando resultados inconclusivos. Entre os estudos avaliados, Chacón J, et al. (2015) constataram que o sexo masculino foi uma variável significativamente associada a menores níveis de adesão terapêutica. Por outro lado, Khayyat SM, et al. (2017) concluíram em seus estudos que o sexo feminino se apresentou como um fator de risco independente para a baixa adesão terapêutica. Tais inconsistências podem revelar a dificuldade de definir o sexo como parâmetro de avaliação de risco isoladamente, sem uma análise íntegra de todos os aspectos sociais e culturais envolvidos na baixa adesão ao tratamento da HAS.

No que se refere à faixa etária, observa-se uma associação entre a idade mais jovem e a baixa adesão terapêutica. Tal correlação também se confirma em análises voltadas para a população de faixa etária idosa, onde observa-se que os idosos de idade mais jovem apresentam menores níveis de adesão ao tratamento medicamentoso em relação àqueles de idade mais avançada (VARLETA P, et al., 2015).

Isso pode estar relacionado a um menor sentimento de medo acerca das complicações relacionadas à HAS em pacientes mais jovens, sobretudo pelo fato de a enfermidade apresentar, em grande parte dos casos, um ritmo de evolução insidioso; a maioria das manifestações sintomatológicas da HAS são relacionadas ao acometimento crônico dos órgãos alvo, logo, apresentam-se mais frequentemente em indivíduos de idade mais avançada e com maior tempo de evolução da doença.

As complicações tardias, isto é, anos após o diagnóstico, podem gerar um menor imediatismo na busca de tratamento adequado para a doença e uma falsa sensação de cura nos pacientes de idade mais jovem (AIOLFI CR, et al., 2015; CUMMINGS, DM et al., 2016; KHAYYAT SM, et al., 2017; VARLETA P, et al., 2015).

Outro fator sociodemográfico associado a menor adesão ao tratamento é o vínculo empregatício. A alta carga horária trabalhista, bem como o estresse psicossocial relatado pelos trabalhadores, são fatores que impactam negativamente o nível de adesão ao tratamento farmacológico da HAS. Isso demonstra uma maior necessidade da elaboração de estratégias de apoio e estímulo à adesão medicamentosa direcionadas para a classe dos trabalhadores (VARLETA P, et al., 2015).

Por fim, a relação entre o apoio social e a adesão ao tratamento é amplamente investigada e diversos estudos relatam uma forte associação entre essa variável e o nível de adesão medicamentosa no tratamento da HAS. A falta de apoio familiar e social apresenta-se como um impasse para adesão terapêutica de HAS, sobretudo em pacientes idosos. Logo, é importante que o círculo social do portador de HAS esteja envolvido e atuando com suporte e estímulo para uma eficaz adesão ao tratamento (NASHILONGO MM, et al., 2017; RESENDE AKM, et al., 2018). 


\section{Fatores associados a condições clínicas, hábitos de vida e outras características intrínsecas aos pacientes}

A adesão ao tratamento anti-hipertensivo mostrou-se ser influenciada por condições particulares de cada paciente, como fatores psicológicos, estilo de vida e comorbidades. Esses elementos apontam a importância da conscientização e autorresponsabilização do paciente em seu processo terapêutico, bem como evidenciam possíveis alvos de intervenções que possibilitem uma melhor adesão ao tratamento.

Os estudos demonstram que, dentre os fatores psicológicos, o estresse, as alterações no padrão de sono, a depressão e a baixa autoeficácia destacam-se como elementos que influenciam negativamente a adesão ao tratamento farmacológico da HAS. De fato, sabe-se que condições deletérias à saúde mental podem interferir negativamente nos hábitos de autocuidado em pacientes portadores de HAS e, portanto, devem ser sempre pesquisadas durante as consultas de acompanhamento (AL-NOUMANI H, et al., 2017; CHACÓN J, et al., 2015; MOURA, AA et al., 2016).

Nessa perspectiva, Al-Noumani $\mathrm{H}$, et al. (2017) defendem que a autoeficácia consiste em um fator indispensável para a criação e manutenção de comportamentos positivos em saúde, como a adesão à terapia medicamentosa. Isso se dá porque tal característica psicológica denota o grau de confiança que o indivíduo deposita na realização de uma dada atividade.

Outro ponto importante foi mencionado por Moura AA, et al. (2016) ao associar emoções e afetos de cunho negativo ao desenvolvimento de estresse, hostilidade, raiva, além de culpa, vergonha e medo. Em vista disso, reitera-se a influência de fatores emocionais e psicológicos no estabelecimento do autocuidado em saúde, o que inclui a adesão à medicação.

Outros fatores de cunho psicológico que também se encontram associados à baixa adesão terapêutica são as percepções errôneas acerca da HAS, das suas complicações e da importância do tratamento, bem como o medo de interações medicamentosas. Esses fatores podem ser abordados pela equipe de saúde através de intervenções de educação em saúde e consultas mais esclarecedoras e voltadas à realidade dos pacientes atendidos (AL-NOUMANI H, et al., 2017; LULEBO AM, et al., 2015; PÉREZ R, et al., 2016; RESENDE AKM, et al., 2018).

No que diz respeito à associação entre comorbidades crônicas e não adesão ao tratamento da HAS, os artigos que compõem este trabalho divergem em seus resultados. Enquanto Khayyat SM, et al. (2017) e demonstraram que a ausência diabetes mellitus estava associada a uma menor adesão à terapêutica, Schoenthaler A, et al. (2017) concluíram que a presença dessa doença atua como fator de risco para a não adesão ao tratamento anti-hipertensivo. Esses resultados conflitantes podem ser fruto, dentre outros fatores, da oposição entre a dificuldade em gerenciar o tratamento de múltiplas doenças e o maior cuidado com a saúde gerado pela experiência no manejo pessoal da doença preexistente.

Evidencia-se também que fatores associados aos hábitos de vida dos pacientes podem sugerir uma maior propensão à aderência medicamentosa irregular. A título de exemplo, Rocha MLF, et al. (2017) identificaram o uso de bebidas alcoólicas e a alimentação rica em gorduras ou frituras como fatores de risco para baixa adesão terapêutica.

Similarmente, Moura AA, et al. (2016) relataram que a dificuldade de adaptação a mudanças no estilo de vida requeridas para um adequado controle dos níveis pressóricos foi um fator intimamente relacionado à baixa adesão medicamentosa.

Algumas características do próprio ato de medicar-se também correspondem a importantes elementos que podem propiciar a não adesão. A utilização de múltiplos medicamentos ou doses em um mesmo dia, a dificuldade de ler, abrir ou listar os medicamentos, bem como o esquecimento são fatores comumente reportados pelos pacientes que prejudicam a adesão medicamentosa.

Esses fatores também podem ser alvo de ações da equipe de saúde com foco em prescrições acessíveis à realidade do paciente e do serviço, bem como no uso de estratégias de lembretes (i.e. mensagens de texto) para possibilitar o uso correto dos medicamentos (BARRETO MS, et al., 2015; ROCHA MLF, et al., 2017; BECHO AS, et al., 2017; LULEBO AM, et al., 2015; VARLETA P, et al., 2015). 


\section{Fatores associados a unidades e equipes de saúde}

Ao considerar-se que uma parcela significativa da população atendida na atenção primária apresenta um baixo nível socioeconômico e educacional, e que, isso por si só já constitui importante fator de risco para a não adesão terapêutica, é evidente a importância do suporte que deve ser proporcionado a tais pacientes pelas unidades de saúde e pela equipe multiprofissional, sendo essa uma temática abordada por vários dos artigos analisados nesta revisão bibliográfica.

Lulebo AM, et al. (2015) descreveram a falta de educação em saúde para hipertensos nas unidades de saúde como um significativo fator associado a não aderência ao tratamento. A adequada instrução e comunicação com os pacientes pela equipe de saúde está associada a novas percepções acerca da HAS e da considerável relevância do tratamento farmacológico dessa patologia.

Sob esse viés, a adequada relação médico-paciente mostra-se como um importante fator para promover uma adequada aderência ao tratamento. Schoentaler A. et al. (2018) demonstraram, através de um estudo observacional realizado com 75 pacientes hipertensos atendidos em centros de APS, que a decisão clínica compartilhada e a maior duração da relação médico-paciente estavam associadas a uma melhor adesão ao tratamento farmacológico.

Destaca-se que as consultas centradas nos pacientes $e$ as discussões sobre as condições sociodemográficas e sobre o tratamento podem ser significativas para educar os pacientes, compreender as dificuldades enfrentadas pelos mesmos e possibilitar uma adequada adesão ao tratamento (SCHOENTHALER A, et al., 2017).

Ademais, uma grande atenção deve ser concedida à terapia que será adotada para os pacientes. Efeitos adversos do tratamento anti-hipertensivo também foram reportados como relevantes fatores que dificultam a aderência ao tratamento (BECHO AS, et al., 2017; VARLETA P, et al., 2015).

Além de informar cautelosamente aos pacientes sobre a possibilidade de efeitos colaterais dos medicamentos prescritos, é imprescindível que o médico, através de uma anamnese apropriada, reconheça nas consultas de acompanhamento se o tratamento está ocasionando prejuízos na qualidade de vida do paciente e, conforme necessário, avalie a necessidade de modificações no esquema terapêutico vigente.

É válido ressaltar, todavia, que algumas das barreiras ao adequado manejo da hipertensão arterial relacionados às unidades de saúde independem do desempenho da equipe profissional. A título de exemplo, a falta de medicações nos postos de saúde também é um preocupante fator que dificulta o controle da HAS, uma vez que os pacientes hipertensos que não encontram as medicações disponíveis nos postos estão duas vezes menos propensos a aderirem ao tratamento (LULEBO AM, et al., 2015). Os conflitos de agendamento também foram reportados como possíveis barreiras à adesão terapêutica, uma vez que prejudicam a acessibilidade aos serviços de saúde (HERSKIND J, et al., 2020).

\section{CONSIDERAÇÕES FINAIS}

Percebe-se a existência de uma grande variedade e heterogeneidade de fatores que podem exercer um impacto negativo na adesão à terapia farmacológica em pacientes portadores de HAS, o que se releva como um importante impasse para o controle da enfermidade e para a prevenção das severas complicações secundárias à mesma. No contexto da APS, a identificação de tais fatores mostra-se extremamente relevante para possibilitar o planejamento e execução de intervenções efetivas, reforçando a necessidade de exercer o cuidado em saúde de forma holística e centrada no paciente, em sua individualidade e contexto social.

\section{REFERÊNCIAS}

1. AlOLFI, CR, et al. Adesão ao uso de medicamentos entre idosos hipertensos. Revista Brasileira de Geriatria e Gerontologia, 2015; 18(2): 397-404.

2. AL-NOUMANI, H, et al. Relationship Between Medication Adherence and Health Beliefs Among Patients with Hypertension in Oman: Pilot study. Sultan Qaboos University medical journal, 2017; 17(3): e329-e333.

3. BARRETO MS, et al. Prevalência de não adesão à farmacoterapia anti-hipertensiva e fatores associados. Revista Brasileira de Enfermagem, 2015; 68(1): 60-67.

4. BECHO AS, et al. Dificuldades de adesão ao tratamento por hipertensos de uma unidade de atenção primária à saúde. Revista de APS - Atenção Primária à Saúde, 2017; 20(3): 349-359. 
5. CHACÓN J, et al. Evaluación del control de la presión arterial y la adherencia terapéutica en hipertensos seguidos en el programa de salud cardiovascular (PSCV): asociación con características clínicas, socioeconómicas y psicosociales. Rev. chil. cardiol, 2015; 34(1): 18-27.

6. CORREIA BR, et al. Perfil Clínico-Epidemiológico de Pacientes Assistidos em Clínicas de Hipertensão. Journal of Health Sciences, 2017; 19(2): 171-176.

7. CUMMINGS DM, et al. Perceived Social Standing, Medication Nonadherence, and Systolic Blood Pressure in the Rural South. The Journal of rural health : official journal of the American Rural Health Association and the National Rural Health Care Association, 2016; 32(2): 156-163.

8. DALLACOSTA FM, et al. Adesão ao tratamento e hábitos de vida de hipertensos. Revista de Pesquisa: Cuidado é Fundamental. (Online), 2019; 11(1): 127-131.

9. DANTAS RCO, RONCALLI AG. Protocolo para indivíduos hipertensos assistidos na Atenção Básica em Saúde. Ciência e Saúde Coletiva, 2019; 24(1): 295-306.

10. GEWEHR DM, et al. Adesão ao tratamento farmacológico da hipertensão arterial na Atenção Primária à Saúde. Saúde em Debate, 2018; 42(116):179-190.

11. HERSKIND J, et al. The outpatient management of hypertension at two Sierra Leonean health centres: A mixedmethod investigation of follow-up compliance and patient-reported barriers to care. African Journal of Primary Health Care; Family Medicine, 2020; 12(1): 1-7.

12. JORNADA BA, et al. Teste de Morisky-Green e Brief Medication Questionnaire para avaliar adesão a medicamentos. Revista de Saúde Pública, 2012; 46(2): 279-289.

13. KHAYYAT SM, et al. Predictors of Medication Adherence and Blood Pressure Control among Saudi Hypertensive Patients Attending Primary Care Clinics: A Cross-Sectional Study. PloS one, 2017; 12(1): e0171255.

14. LULEBO AM, et al. Predictors of non-adherence to antihypertensive medication in Kinshasa, Democratic Republic of Congo: a cross-sectional study. Biomedcentral Research Notes, 2015; 8: 526.

15. MAGRINI DW, MARTINI JG. Hipertensão arterial: principais fatores de risco modificáveis na estratégia saúde da família. Enfermería Global, 2012; 11(26): 344-353.

16. MALACHIAS MVB, et al. $7^{\underline{a}}$ Diretriz Brasileira de Hipertensão Arterial. Arquivos Brasileiros de Cardiologia, 2016; 107(Supl.3): 1-83.

17. MOURA AA, et al. Factores de no adhesión al tratamiento de la hipertensión arterial. Revista Enfermeria Global, 2016; 15(43): 1-13.

18. NASHILONGO MM, et al. Assessing Adherence to Antihypertensive Therapy in Primary Health Care in Namibia: Findings and Implications. Cardiovascular drugs and therapy, 2017; 31 (5-6): 565-578.

19. PÉREZ-ROSABAL E, et al. Adherencia terapéutica y creencias sobre su salud en pacientes hipertensos. Medisan, 2016; 20(1).

20. REINERS AAO, et al. Adesão ao tratamento de hipertensos da atenção básica. Ciência, Cuidado e Saúde, 2013; 11(3): 581-587.

21. RESENDE AKM, et al. Dificuldades de idosos na adesão ao tratamento da hipertensão arterial. Revista de Enfermagem da UFPE on line, 2018; 12(10): 2546-2554.

22. ROCHA MLF, et al. Adesão ao tratamento da hipertensão arterial entre usuários da estratégia saúde da família em um município do Piauí. Revista de APS, 2017; 20(1): 6-20.

23. SILVA MLB, BOUSFIELD ABS. Representações sociais da hipertensão arterial. Temas de Psicologia, 2016; 24(3): 895-909.

24. SCHOENTHALER A, et al. Addressing the Social Needs of Hypertensive Patients: The Role of Patient-Provider Communication as a Predictor of Medication Adherence. Circulation. Cardiovascular quality and outcomes, 2017; 10 (9).

25. SCHOENTHALER A, et al. Medication Adherence Improvement Similar for Shared Decision-Making Preference or Longer Patient-Provider Relationship. Journal of the American Board of Family Medicine: JABFM, 2018; 31(5): 752760.

26. VARLETA $P$, et al. Prevalencia y determinantes de adherencia a terapia antihipertensiva en pacientes de la Región Metropolitana. Revista Médica de Chile, 2015; 143(5): 569-576. 